Revue d'histoire de l'Amérique française

REVUE D.HISTOIRE DE L'AMÉRIQUE FRANÇAISE

\title{
La Mennais et l'éducation au Bas-Canada
}

\section{Thomas Matheson}

Volume 13, numéro 4, mars 1960

URI : https://id.erudit.org/iderudit/302003ar

DOI : https://doi.org/10.7202/302003ar

Aller au sommaire du numéro

Éditeur(s)

Institut d'histoire de l'Amérique française

ISSN

0035-2357 (imprimé)

1492-1383 (numérique)

Découvrir la revue

Citer cet article

Matheson, T. (1960). La Mennais et l'éducation au Bas-Canada. Revue d'histoire de l'Amérique française, 13(4), 476-491. https://doi.org/10.7202/302003ar d'utilisation que vous pouvez consulter en ligne.

https://apropos.erudit.org/fr/usagers/politique-dutilisation/ 


\title{
LA MENNAIS ET L'ÉDUCATION AU BAS-CANADA
}

\begin{abstract}
Avant d'écrire l'histoire des idées, les chercheurs doivent établir les faits historiques eux-mêmes et évaluer exactement leur portée. L'évolution de la pensée, à l'intérieur de toute société humaine, est intimement liée à la succession même des événements. Les hommes n'ont pas d'idées innées.

Brunet, Trois dominantes de la pensée canadiennefrançaise.*
\end{abstract}

Le 9 mars 1816, dans la vieille cité bretonne de Vannes, Hugues-Félicité-Robert de La Mennais, tremblant d'émotion, d'hésitation, reçoit les ordres. Certes il a toute la ferveur d'un néophyte, mais il ignore ce que ce geste aura de répercussions dans le monde. Il se veut humble; par deux fois son verbe retentira à travers toute l'Europe et l'Amérique. L'Amérique ..., il veut s'y consacrer, s'y épanouir en servant Dieu. ${ }^{1}$ La Providence y pourvoit autrement. Son ami, le sulpicien Teysseyre le pousse à écrire une réfutation des thèses de Jean-Jacques Rousseau afin de l'occuper. Rêve-t-il encore de l'Amérique ? Ses pieds ne fouleront jamais ce sol mais sa pensée, son Essai sur l'Indifférence en matière de religion y créera toute une commotion. Par qui ? Par un autre sulpicien, l'abbé Jean-Jacques Lartigue.

Parti le 3 juillet 1819, de Québec, l'abbé Lartigue alla en Angleterre en compagnie de Mgr Joseph-Octave Plessis, évêque de Québec, et de l'abbé Pierre-Flavien Turgeon, son secrétaire intime. Ce fut un voyage très important pour les trois. Mgr Plessis voulait visiter Rome en vue de diviser son diocèse qui était alors aux dimensions d'un continent. Lartigue, alors au Séminaire de Montréal, allait à Londres, en qualité d'avocat, en vue de défendre les biens de la seigneurie de Saint-Sulpice. ${ }^{2}$

* M. Brunet, Trois dominantes de la pensée canadienne-française: l'agriculturisme, l'anti-étatisme et le messianisme \$, dans Ecrits du Canada français, III: (1957) 33.

1 F. Duine, La Mennais, sa vie, ses idées, ses ouvrages, 38.

2 Brasseur de Bourbourg, Histoire du Canada, de son église et de ses missions, II : 157-160. A propos de Bourbourg cf.: Robert Sylvain, La vie et l'cuvre de Henry de Courcy (1820-1861), (Québec PUL, 1955), 197-202. 
Par ses relations anglaises, l'abbé Lartigue entendit probablement parler d'un volume qui avait grande vogue sur le continent. Le livre lu, l'auteur l'impressionne. Il note dans son journal de voyage: "Quel terrible homme contre l'impiété est ce $\mathbf{M}^{r}$ de la Mennais, dont j'ai commencé à lire l'ouvrage contre l'Indifférence en fait de Religion! Cet ouvrage, par l'énergie de ses pensées, la profondeur de ses raisonnements, par la force de son élocution, est digne de faire époque dans les annales du Christianisme. $\gg^{3}$

Mgr Plessis de son côté entre en contact avec le milieu spirituel dans lequel évoluera pendant quelques années celui que Victor Cousin a appelé «cet incendiaire de la pensée ». Il est piloté, coïncidence heureuse, par l'abbé Philippe-Jean-Louis Desjardins, frère de Louis-Joseph, son premier curé à la cathédrale de Notre-Dame de Québec.

Curieuse histoire, celle de ces deux frères, malheureuse aussi. Tous deux ont fui la rage jacobine et la terreur révolutionnaire. Après un séjour en Angleterre, ils sont venus s'installer au Canada en 1794. Philippe est venu le premier, en 1793, à la tête d'une mission destinée à préparer l'établissement en Canada de plusieurs de ses compatriotes ecclésiastiques. Il vient à la demande de Mgr Hubert qui, à l'époque, voyait son clergé incapable de répondre aux besoins religieux de la population. ${ }^{4}$ Par un soudain retour des choses qu'on s'explique aujourd'hui, les autorités coloniales anglaises ont facilité ces projets. L'aimable réception du lieutenant gouverneur Alured Clarke, le 3 mars 1793, au Château St-Louis, cachait en réalité un dessein intéressé. Les prêtres royalistes émigrés serviraient à répandre l'aversion pour la France athée et jacobine; et l'on contrecarrait ainsi la propagande républicaine des Etats-Unis parmi la population des Canadas. ${ }^{5}$

3 APQ., Lartigue, Journal, 30 juin 1819 - 7 août 1820, 156, date 18 janvier 1820, cité dans Ouellet, Mgr Plessis et la naissance d'une bourgeoisie canadienne (1797-1810), dans Société canadienne d'histoire de l'Eglise catholique, rapport $1955-56,98$.

4 N.-E. Dionne, Les ecclésiastiques et les royalistes français réfugiés au Canada d̀ l'époque de la révolution, 1791-1802,65.

5 M. G. Hutt, Abbe P. J. L. Desjardins and the scheme for the settlement of French priests in Canada, 1792-1802, dans Canadian Historical 
Aidé par la rencontre de desseins assez différents de part et d'autre, l'abbé Philippe Desjardins put faire venir son frère l'abbé Louis-Joseph. Dans les années qui suivirent, au moins quarante-cinq émigrés ecclésiastiques passèrent au Canada, parmi lesquels Jacques-Ladislas-Joseph de Calonne sur qui nous reviendrons plus loin. Louis-Joseph, envoyé d'abord en mission dans la Baie des Chaleurs, est nommé vicaire à Notre-Dame de Québec, en 1801. Là, il a pour curé Mgr Plessis, coadjuteur de

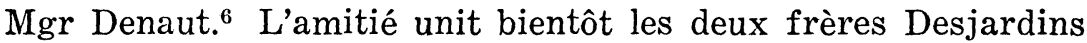
et le prélat. L'abbé Philippe rentre en France en 1802. Il reste pourtant en correspondance avec Mgr Plessis qui devient en 1806 évêque de Québec.7

En son voyage vers Rome, Mgr Plessis avait éprouvé une «joie aussi sincère que légitime ». Il rencontrait à Paris l'ami dont la vie l'avait séparé depuis 17 ans. Devenu grand-vicaire de l'évêque d'Orléans, ${ }^{8}$ l'abbé Philippe introduit Mgr Plessis dans les milieux royalistes. L'évêque de Québec est reçu par Louis XVIII et le comte d'Artois. «Il va chez l'abbé Barruel qui lui monte la tête sur la franc-maçonnerie, au point qu'après avoir enregistré minutieusement l'entretien il concède qu'il peut $\mathbf{y}$ avoir un peu de système. ${ }^{9}$ En Savoie, à un dîner, «il rencontre le comte de Maistre, ci-devant ambassadeur de Sa Majesté Sarde à Petersbourg, et maintenant secrétaire d'État à Turin. Cet homme instruit, bon catholique et d'une conversation aimable, publie pendant sa légation de Russie, un ouvrage sous le titre de Considérations sur la France qui fut bien accueilli par le public et lui a donné de la réputation. Il venait d'en publier un autre ayant pour titre: Du Pape. ${ }^{10}$ Viatte souligne: «à la

Review, vol. XXXIX: no 2, (1958), 93-124; il cite Stevenson to Simcoe, April 27, 1793, and to Dundas, July 1793, quoted in E. A. Cruikshank ed., The Correspondence of Lieutenant-Governor, John Graves Simcoe, (5 vols., Toronto, 1923-31), I: 319, 410.

6 Dionne, op. cit., 211.

$7 \mathrm{Mgr}$ Henri Têtu, Journal d'un voyage en Europe de Monseigneur Plessis (Québec, 1905), 75.

8 Têtu, op. cit., 74; Dionne, op. cit., 182, écrit «grand vicaire de Sainte Geneviève 》.

9 A. Viatte, Histoire littéraire de l'Amérique française, des origines à nos jours 1950 (Paris, PUF, 1954), 52.

10 Têtu, op. cit., $357 \mathrm{~s}$. 
façon dont il note ce qu'est l'homme et son œuvre antérieure, on a d'ailleurs l'impression qu'il les ignorait jusqu'alors ».11

A son retour de Rome, Mgr Plessis qui a obtenu la division de son diocèse, est impressionné par un sermon de l'abbé Frayssinous à l'église Saint-Sulpice, «une de ces savantes conférences qui, depuis quelques années, l'ont mis en si grande réputation, et ont ramené à la vraie foi un si grand nombre d'esprits égarés par les fausses lueurs du philosophisme. Le sujet de celle-ci était la Résurrection de Jésus-Christ, prouvée par des arguments dont chacun portait, avec lui, la conviction la plus irrésistible. L'abbé Frayssinous parle avec l'autorité d'un docteur qui sait bien que ses raisonnements sont sans réplique. Il ne dissimule pas les objections; il les présente dans toute leur force, mais en même temps il les met en poudre. ${ }^{12}$

Mgr Plessis, heureux, certes, d'un voyage aussi agréable que profitable, rentre à Londres. De là il transmet à Jean-Jacques Lartigue le bref apostolique qui le nomme évêque de Telmesse et administrateur du district de Montréal. Les deux prélats reviennent au Bas-Canada avec la secrète ambition de faire connaître leurs relations et leurs lectures.

Mais ... désillusion ! La renommée de l'auteur dont l'ouvrage les a enthousiasmés, a déjà traversé l'Atlantique par on ne sait quelle voie. En effet, à la fin de l'année 1819, une controverse s'est élevée autour du système d'enseignement mutuel, (méthode dite de Lancaster). L'abbé Jacques-Ladislas-Joseph de Calonne est entré en lice contre le système, armé d'une page de l'Essai sur l'Indifférence en matière de religion.

Cet abbé de Calonne, frère du ministre de ce nom sous Louis XVI, et «l'un des débris les plus respectables de l'ancien clergé français »,13 est débarqué un jour mystérieusement sur l'Île-du-Prince-Édouard, alors Île Saint-Jean. Après un séjour en Angleterre, il arrive à Québec, le 21 octobre 1807 et se rend au chapelinat des Ursulines des Trois-Rivières que Mgr Plessis

11 Viatte, op. cit., 52.

12 Têtu, op. cit., 409.

13 Bourbourg, op. cit., II : 119. 
lui a confié. ${ }^{14}$ C'est à ce poste qu'il dut recevoir un des compagnons de Joseph Lancaster. Ce dernier, un londonien avait adopté le système d'enseignement apporté en Angleterre en 1797 par l'écossais Andrew Bell, système alors en usage à Madras, aux Indes. Ce système, connu sous le nom de Madras System, garda le nom de méthode lancastrienne bien que Lancaster ne l'ait pas inventée. Le compagnon de Lancaster demanda l'appui de l'abbé de Calonne. L'abbé refusa. Une école s'établit quand même, dirigée par Silby Burn des Trois-Rivières. ${ }^{15}$ Alors l'abbé de Calonne, fort du témoignage de La Mennais, ${ }^{16}$ écrivit aux journaux, la Gazette des Trois-Rivières et la Gazette de Québec:

Mr le Rédacteur,

Je vois que des Sociétés de Londres, montrent beaucoup de zèle, et se donnent beaucoup de mouvemens, pour procurer de l'éducation aux jeunes canadiens. Ce zèle qui se porte si loin, a-t-il pour but de nous donner des principes et des mœurs? J'avoue que je n'eus jamais pensé que deux articles si essentiels, nous fussent venus de Londres. Quelques-uns de ceux qui nous ont été envoyés pour conduire cette entreprise, m'ayant fait l'honneur de me faire part de leur projet, je leur fis franchement quelques objections, auxquelles ils répondirent aussi franchement, que dans leurs écoles on ne parloit point de Religion.

Ayez la bonté, Monsieur, d'insérer dans votre feuille les brièves reflexions suivantes. Elles sont tirées d'un ouvrage très récent et très célèbre en France. J'ose dire que Bossuet s'en seroit fait honneur. Ce livre est intitulé: «De l'indifférence en matière de Religion》. Avant que le second volume paroisse, le premier a été réimprimé quatre fois en un an, au nombre de douze mille exemplaires chaque fois.

14 Dionne, op. cit., 311.

15 L.-P. Audet, Le Système Scolaire de la Province de Québec, - IV L'Institution Royale, le déclin; 1825-1846 (Québec, PUL, 1952), 234.

16 \& Il y a conflit entre les historiens, autour de l'orthographe de ce mot. Le clergé (Ladoue, Ricard, Boutard, Duine) adopte ménaisien, les laĩques (Maréchal, Feugère, etc.) trouvent plus naturel d'écrire mennaisien. : Henri Brémond, dans sa biographie de Gerbet (Paris, Bloud, 1907, XI371 p.), adopte l'orthographe de Maréchal. 
Voici les réflexions de cet auteur, page 500 :

$\mathrm{Ne}$ l'oublions jamais, la religion est l'unique éducation du peuple. Sans la Religion il ne sauroit rien, rien sur tout de ce qu'il importe le plus à la société qu'il sache et à lui de savoir. Il ignoreroit également et les devoirs de l'homme et sa destinée. Il végéteroit au milieu des Académies, des Universités, des Gymnases, dans un abrutissement, cent fois pire que l'état sauvage. La Religion le civilise; elle nourrit le pauvre de vérités comme elle le nourrit de pain; elle éclaire, elle agrandit son intelligence; et le dernier des petits enfans instruits à son école, plus véritablement philosophe qu'aucun des prétendus sages qui ne reconnoissent d'autre guide que leur raison, confondroit, le Catéchisme à la main, cette raison altière, par la sublimité de ses enseignements. Il étoit digne d'un philosophe matérialiste, en substituant les évolutions à des instructions, et en mettant entre les mains une pierre muette, en place du livre où il puisoit ces hautes et importantes leçons ...

J'ai l'honneur d'être, Monsieur

Votre très-humble,

De Calonne, Prêtre

«L'auteur parle ici des Écoles Lancastriennes qui, malgré les cris et les efforts de nos soi-disant philosophes, n'ont pu s'introduire, presque nulle part en France. Il en sera de même ici. Elles crouleront avant d'avoir pu se glisser dans aucune de nos Campagnes. » ${ }^{17}$

L'abbé ne cache pas son enthousiasme à l'égard de La Mennais. Avec ses contemporains français il classe l'écrivain parmi les Pères de l'Église. Mais son enthousiasme pour l'homme il n'en a rien dit dans les journaux. Il ne l'a pas même nommé. Or voici que, dans la Gazette de Québec, 22 novembre, un ardent partisan du système de Lancaster rétorque que «l'auteur François de M. de Calonne contribueroit sans doute avec joie à

17 La Gazette de Québec, 15 novembre 1819. Nous avons fouillé la Gazette des Trois-Rivières, dirigée par Ludger Duvernay. Nous n'avons pas trouvé le numéro dans lequel Calonne publia cette lettre, le numéro manquant probablement. La Gazette des Trois-Rivières existe sous microfilm à la Bibliothèque de la Législature de Québec. 
l'avancement de la même bonne œuvre en France ». Singulière riposte. Fervent disciple de La Mennais, l'abbé de Calonne n'a pas suivi les journaux auxquels son auteur favori collabore en France. Sans quoi il aurait pu répondre à son contradicteur que, précisément dans le Conservateur, journal fondé par Chateaubriand, l'auteur de l'Essai sur l'Indifférence venait d'écrire un article sur l'«Éducation du peuple». Et sa conclusion était précisément celle-ci : «adopter la méthode d'enseignement mutuel (dite méthode lancastrienne), c'est donner sa préférence à l'anarchie $»^{18}$

Ainsi La Mennais entrait incognito dans les polémiques canadiennes et sur quel sujet ? L'éducation. En ce domaine il n'allait pas rester longtemps inconnu.

Le 22 novembre 1810, Mgr Plessis accorde la permission à l'abbé Antoine Girouard, curé de Saint-Hyacinthe depuis 1805, de fonder une maison d'éducation supérieure. Mais il ajoute: « Je ne voudrais pas cependant que ce nouveau-né fit tort à son grand frère qui n'est encore qu'au commencement de son adolescence. » ${ }^{19}$ Ainsi naît une rivalité entre le séminaire de Nicolet et celui de Saint-Hyacinthe. Qu'aurait pensé Mgr Plessis s'il avait pu prévoir qu'en ce nouveau Séminaire, un mouvement doctrinal se développerait dont les répercussions s'étendraient à tout le $\mathrm{XIX}^{\mathrm{e}}$ siècle? Nous voulons dire l'adhésion de plusieurs professeurs au libéralisme catholique formulé au début par La Mennais, puis repris, en quelque façon, par Lacordaire et Montalembert.

En effet, le premier janvier 1824, Mgr Plessis donnait pour supérieur au Séminaire de Saint-Hyacinthe, le premier disciple canadien de La Mennais, Mgr Jean-Jacques Lartigue. Par ses relations sulpiciennes à Paris, ce dernier est bien au courant des publications de La Mennais. Le 15 décembre 1823, il demande à l'abbé Jean-Baptiste Thavenet de lui faire parvenir un certain nombre de livres. Et il lui fait observer qu'il n'a pas reçu le

18 Duine, op. cit., 68. I : 23 .

19 Chanoine C.-P. Choquette, Histoire du Séminaire de Saint-Hyacinthe, 
Drapeau blanc depuis la mi-juillet.. ${ }^{20}$ L'abbé Lartigue ignorait donc l'attaque de l'auteur de l'Essai contre l'Université et contre Frayssinous, attaque qui valut au journal une condamnation judiciaire.

En sa qualité de supérieur, l'évêque de Telmesse nomme, à ce qu'il semble, vers la fin de juillet 1831, l'abbé Jean-Charles Prince, directeur du Séminaire de Saint-Hyacinthe. Ce dernier a fait ses études au collège de Nicolet; il y a enseigné les BellesLettres en 1823; il exerce, depuis son ordination, le 23 septembre 1826, les fonctions de chapelain de l'église Saint-Jacques et de secrétaire de Mgr Lartigue. ${ }^{21}$ Il partage l'enthousiasme de son évêque pour les doctrines de l'auteur de l'Essai sur l'Indifférence; cet enthousiasme, il le communique même à son plus brillant élève de philosophie, le jeune Joseph-Sabin Raymond. Entre les deux abbés une correspondance s'échange vers 1830 à ce sujet. M. Raymond est conquis, à tel point que l'élève menace de dépasser bientôt le maître: — « Excuse mon brave petit Lamennais... Est-ce de St-Hyacinthe qu'originait cet anonyme, qui tapait, l'autre jour l'Éditeur de La Minerve à propos de la confrontation de ses principes avec ceux de Lamennais? ça m'en a tout l'air... La cause de Lamennais triomphe et c'est la bonne cause aux yeux de tous les amis de ce qui est bon et religieux. ${ }^{22}$

Les deux abbés gagnèrent à la même cause le professeur de philosophie, l'abbé Isaac-Stanislas-Lesieur Désaulniers. Deux ans plus tard, dans un article à La Minerve, l'abbé Prince écrit:

20 Le Drapeau blanc, fondé en 1819 par Martinville, était un organe du parti ultra-royaliste. Parmi les rédacteurs, on remarque Charles Nodier. L'abbé Thavenet, naguère émigré au Canada, était à Paris depuis 1815. Il avait pour mission de recueillir les rentes des communautés religieuses canadiennes, succursales des communautés françaises. Il faisait affaire par l'intermédiaire des libraires, à Paris Méquignon, à Londres maison Moreland, Duckett \& Co et à Québec MM. Paterson et Young, cf. Dionne, op. cit., 229 - Lartigue à Thavenet, directeur du séminaire de St-Sulpice, à Paris (Montréal, 15 décembre 1823), RAPQ, (1941-42), 433.

21 Choquette, op. cit., I: 127.

22 Ibid., I: 128-129. Nous n'avons pas retrouvé cet article. Choquette ne cite pas ses sources et il doit faire erreur en prétendant que dans les lettres de novembre 1830, \& M. Prince s'employa avec ardeur à gagner M. Raymond à la cause des (Pèlerins de Rome et de la liberté) 》. La Mennais partira pour Rome le 21 novembre 1831 . Tout au plus Prince connaissait-il l'existence du journal l'Avenir. 
* Oui, monsieur, nous enseignons du La Mennais, non pas son système politique, mais bien sa thèse philosophique: c'est-à-dire que nous combattons le sens privé des Déistes, des Athées, etc., par le sens commun des hommes ou l'autorité du genre humain, tenant toujours bon compte des autres preuves philosophiques qui les refutent, mais les basant toutes sur celles-là. ${ }^{23}$

Curieuse histoire que celle de cet enseignement philosophique.

Rappelons-nous que le second volume de l'Essai parut en juillet 1820. Boutard, qui le résume, commente: «L'apparition du deuxième volume de l'Essai sur l'indifférence allait marquer l'entreprise la plus audacieuse qui eut été tentée depuis Descartes. ${ }^{24}$ La Mennais se propose de «montrer que la raison individuelle, ou la raison de chaque homme pris à part, n'est point infaillible. Nos trois moyens de connaître, à savoir les sens, le sentiment, le raisonnement, peuvent nous conduire à la vérité, sans doute, mais ils sont impuissants à nous donner la certitude. L'infaillibilité qui constitue cette dernière n'appartient qu'à la raison générale, c'est-à-dire à l'autorité du genre humain. Un individu ne peut pas dire: «Je suis certain, parce que je vois avec méthode», mais «je suis certain parce que je crois avec l'humanité ». Ainsi la philosophie ménaisienne est une philosophie de la foi, ou de l'autorité, ou du sens commun. Elle s'oppose au cartésianisme, *philosophie aussi dangereuse qu'elle est niaise », philosophie des rationalistes et des athées, des déistes et des hérétiques, qui s'attachent tous au triomphe du sens privé et qui mettent la certitude dans une perception claire et distincte des choses, c'est-à-dire dans l'évidence d'une conviction personnelle $\gg^{25}$

Dans les collèges classiques en voie de s'établir un peu partout dans la province du Bas-Canada, l'enseignement dépendait, à

${ }^{23}$ La Minerve, 26 septembre 1833, cité dans Chanoine Michel Couture, Le mouvement mennaisien au Canada français (1830-1850), dans la Société canadienne d'histoire de l'Eglise catholique, rapport 1939-40, 74. Cf. Matheson, Un pamphlet politique au Bas-Canada: Les Paroles d'un Croyant de La Mennais, thèse à Laval, 56-57.

24 Abbé Charles Boutard, Lamennais, sa vie et ses doctrines (1905), cité dans Dominique Bagge, Les idées politiques en France sous la Restauration (Paris, PUF, 1952), 219.

25 Duine, op. cit., 84 . 
vrai dire, de l'autorité de chaque institution. Les programmes, les manuels quand il en existait, différaient d'une institution à l'autre. Rien de surprenant donc si l'on constate, au début de l'histoire de ces maisons d'enseignement classique, un flottement d'opinion concernant ces problèmes de philosophie. Pour ce qui est des manuels, disons pourtant que, vers les années 1820 et 1830 , l'on déploya un véritable effort pour doter le pays de livres scolaires. " $\mathrm{Au}$ Collège de Montréal, des professeurs composent, vers 1824, et même un peu avant, des Morceaux choisis de divers auteurs pour servir de thème aux écoliers de rhétorique où ils font entrer tout le moderne d'alors: du Mirabeau, du Maury, du Chateaubriand, du Bonald, du Joseph de Maistre, du La Mennais et voire du Jean-Jacques Rousseau. ${ }^{26}$

En 1832, le problème spécial de l'enseignement de la philosophie se pose. L'abbé Désaulniers part à Georgetown, aux Etats-Unis, en vue d'y conquérir une maîtrise ès-arts; « on lui conseille comme auteur de philosophie, le petit ouvrage de l'abbé

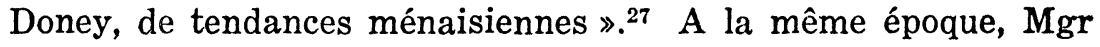
Lartigue se pose le problème d'une autre façon: «si l'on avait des livres élémentaires en nombre suffisant pour enseigner la philosophie de Lamennais, je l'introduirais volontiers $\$ .^{28}$

Heureux de l'assentiment de son supérieur, l'abbé Raymond, ordonné prêtre le 22 septembre 1832, remplace Désaulniers et donne des cours à ses élèves à l'aide du système de La Mennais sur le fondement de la certitude. A la fin de l'année 1833, lors des examens publics, les élèves discutent les théories de Descartes et de La Mennais. L'accueil fait au débat ressemble à celui dont on accable en France la nouvelle philosophie. L'abbé Jacques Odelin, curé de la paroisse de Saint-Hilaire, se dresse, ce jour-là même, en grand adversaire de la théorie du sens commun. Il a déjà été professeur à Saint-Hyacinthe en 1813-14; «il n'avait

26 Abbé O. Maurault, Le Petit Séminaire de Montréal, 78, cité dans Lionel Groulx, L'enseignement français au Canada, I: 198s et Le Français au Canada, 94.

27 Choquette, op. cit., I : 152.

28 Loc. cit. 
peut-être pas conservé un souvenir plaisant de ce séjour $\gg .^{29} \mathrm{Il}$ profite de l'occasion pour confondre les élèves, à tel point que l'abbé Raymond doit intervenir. La discussion se continue dans les journaux: M. Odelin dans l'Ami du peuple, «journal absolument dévoué au gouvernement», les abbés Prince, Raymond, La Rocque dans l'Écho de St-Charles, sous divers pseudonymes: « Un élève de Collège, Un défenseur du sens commun, Un catholique ».

La philosophie ménaisienne passionne les jeunes professeurs de Saint-Hyacinthe; les idées propagées par le groupe de l'Avenir où figurent des hommes tels que les abbés Gerbet et Salinis, l'économiste Charles de Coux, l'abbé Lacordaire et un tout jeune homme, Montalembert, les enflamment davantage. Liberté de conscience, liberté d'enseignement, liberté de la presse, liberté d'association, autant de thèmes qui exaltent et fouettent les emballements des néophytes canadiens, surtout le thème de la liberté de l'enseignement. L'abbé Prince écrit, à ce propos, un article dans la Minerve. Malheureusement, Mgr Lartigue ne partage pas son avis:

Il sera peut-être utile de faire quelques corrections ... . afin de ne pas être enveloppé dans le blâme que votre homme pourrait encourir pour quelques paroles incorrectes ou trop insolentes qui auraient échappé à lui ou à ses collaborateurs dans l'Avenir. Peut être même aurait-il été préférable de ne pas traiter cette matière délicate dans un temps où l'auteur est en accusation auprès du Saint-Siège avant que celui-ci ait prononcé; et dans un pays comme celui-ci où l'on ne peut pas beaucoup se plaindre que l'enseignement soit gêné, tandis qu'on nous veille de près pour nous trouver en faute. Mais je laisse le tout à votre sagesse en vous donnant carte blanche ... ${ }^{30}$

29 Ibid., I: 162. «Un Critique Canadien de Lamennais》 dans le Bulletin des Recherches Historiques, no 47, 253. Il est intéressant d'y noter l'opinion du chanoine Emile Chartier sur l'abbé Odelin: «Le premier en Canada, écrit-il, il soupçonna et mit à nu le poison subtil caché dans les écrits du trop fameux Lamennais.

30 Choquette, op. cit., I: 135, la date du 1er mai 1832, cf. Rapport de l'Archiviste de la Province de Québec (1942-43), 149, la lettre est datée du 4 juin 1832 . 
Cette carte blanche, l'abbé Raymond essaie de la jouer en 1832. Emballé par les discours de Montalembert et de Lacordaire au sujet de l'École libre, il veut en organiser une sorte de répétition aux exercices de fin d'année. Pour rassurer Mgr Lartigue à l'égard des «pèlerins de Dieu et de la liberté », Raymond écrit:

$\mathrm{Au}$ reste je suis flatté d'avoir à vous communiquer un alinéa d'une lettre de l'abbé Lacordaire qui concerne la doctrine de Lamennais et que je reçois tout à l'instant fort à propos. Il m'écrit de Rome, en date du 25 février 1832. Voici ses propres termes: « Rome ne prononcera aucune condamnation, ni ne manifestera aucune désapprobation à l'égard des doctrines soutenues par l'Avenir, nous en avons acquis la certitude. Mais les Etats Pontificaux sont dans une situation qui donne de telles alarmes qu'il est difficile d'obtenir un examen, et un jugement explicite. » Nous voilà donc considérablement rassurés par cette lettre longue et cordiale de notre nouvel ami. ${ }^{31}$

Malheureusement la répétition n'eut pas lieu en 1832; la sortie des élèves se fit précipitamment, sans exercices publics, le 2 juillet, en raison de l'épidémie de choléra qui sévissait dans la province. Le Procès fut repris l'année suivante dans des circonstances assez particulières et fit beaucoup de tapage. Depuis plusieurs années les autorités du collège de St-Hyacinthe sollicitaient la personnalité juridique. Les difficultés politiques, la crise des subsides, les animosités personnelles, empêchèrent la session législative de suivre son cours. Aux exercices littéraires de 1833 , le collège eut l'honneur de recevoir la visite du gouverneur Aylmer; les élèves répétèrent en sa présence les fameux discours de Lacordaire et de Montalembert. Ce geste, qui « offrait pour le moins autant de piquant que d'opportunité », s'attira les

31 Loc. cit. Mgr Lartigue, en effet, s'inquiétait fort de l'attitude de la Papauté au sujet des positions de l'Avenir. Il s'en ouvrait, par lettre, dès janvier 1832, au Docteur Nicolas Wiseman et lui demandait, en postscriptum, \& ce que Rome pensait des principes de l'abbé $F$. de Lamennais pour lesquels nous ne sentons ici aucune répugnance », (RAPQ (1942-43), 132). Mgr Lartigue ne pouvait mieux trouver à qui s'adresser: La Mennais, durant ce second séjour dans la Ville Eternelle, fut en rapport constant avec le brillant directeur du collège anglais (Duine, op. cit., 161-163). 
éloges de la Minerve, journal qui eut pourtant, ainsi qu'on va voir, des démêlés avec les professeurs du collège. ${ }^{32}$

L'enthousiasme des jeunes professeurs pour les doctrines de l'Avenir et la philosophie du sens commun subit une autre douche froide. Elle vint, cette fois, des autorités romaines. L'encyclique Mirari Vos réprouvait la liberté de conscience comme une « maxime absurde », déclarait la liberté de la presse « funeste et exécrable ». «Enfin le Souverain Pontife, Grégoire XVI, dénonçait les partisans de la séparation de l'Église et de l'État et ceux qui, sous prétexte de travailler au bien commun, s'alliaient à des gens de toute espèce de religion. ${ }^{33}$ Or $L a$ Minerve reproduisit la lettre de soumission du groupe de La Mennais, lettre envoyée au rédacteur de la Gazette de France, le 10 septembre 1832. Le groupe y annonçait que « $l$ 'Avenir, provisoirement suspendu depuis le 15 novembre 1831, ne paraîtra plus et que l'Agence générale pour la défense de la liberté religieuse est dissoute. ${ }^{34}$ Au Canada, il ne semble pas que les mennaisiens se soient inquiétés outre mesure. Dans une de ses lettres d'alors, Mgr Lartigue soutient «qu'aucune proposition tirée de La Mennais et de l'Avenir n'a été condamnée, que le pape n'a exigé aucune rétractation [ce qui nous fait croire que Mgr Lartigue a lu La Minerve], que les douze volumes imprimés de La Mennais et son Mémorial catholique ainsi que les œuvres de Gerbet demeurent intacts; et les arguments du premier sur la certitude du critérium du sens commun aussi bien que l'autorité du pape dans l'Église n'ont aucune flétrissure, ni rien perdu de leur force $\gg^{35}$

32 Groulx, op. cit., I: 202.

33 Duine, op. cit., 170-171.

${ }^{34}$ La Minerve, 12 novembre 1832.

$35 \mathrm{Mgr}$ Lartigue à M. (Pierre) Viau, vicaire général, curé de la Rivière-Ouelle (Montréal, 17 novembre 1832) dans RAPQ, (1942-43), 171. Cette lettre est importante: elle fait voir l'ultramontanisme de l'évêque, ultramontanisme inspiré de La Mennais. Cette question, qui a affecté toute notre histoire religieuse au XIX $\mathrm{X}^{\mathrm{e}}$ siècle, mériterait une étude fouillée. La même lettre démontre également l'adhésion de l'évêque au dogme de l'infaillibilité pontificale, qui sera promulgué plus tard en 1870. Elle nous révèle l'intérêt porté par Lartigue aux cuvres des artisans du renouveau religieux en France. Le périodique, Mémorial catholique, fut fondé par les abbés Gerbet et de Salinis en 1823. La première livraison parut en janvier 1824 et vécut grâce au concours de de Bonald. * Il fut un instrument de défense pour La Mennais (Duine) et comme une lente marche vers l'Avenir (Bagge) 》. 
Toutefois l'adhésion des professeurs de Saint-Hyacinthe au mennaisianisme, adhésion par trop manifeste, et dans les exercices littéraires de 1833, et dans la querelle de la liberté de l'enseignement, et dans l'enseignement de la philosophie du sens commun, subit un rude choc lorsqu'on apprit la publication des Paroles d'un Croyant. Un article de la Minerve déclenche la débâcle du mennaisianisme parmi le clergé canadien. Les Paroles ont provoqué une suite de pastiches. La Minerve publie, par exemple, une critique de la brochure: les Contre-Paroles d'un Croyant, d'Elzéar Ortolan; ce dernier vise les sympathies mennaisiennes du corps professoral de Saint-Hyacinthe:

On a dit que l'écrit qu'il refute a eu, parmi nos jeune (sic) lévites, un retentissement dont de bonnes âmes s'effraient; on a cité même un séminaire fameux où la séduction serait allée jusqu'au point de préparer de fanatiques ovations au grand publiciste en soutane. Eh bien ! s'il en est ainsi, n'étaitce pas le cas d'adresser, comme l'antidote du poison emmiellé, les contre-paroles aux jeunes lévites dont il aurait corrompu la piété $?^{36}$

La réaction ne se fait pas attendre parmi les sympathisants mennaisiens. Dans le Canadien du 29 août 1834 on peut lire cette nouvelle: "Le pape vient d'anathémiser les Paroles d'un Croyant dans une lettre encyclique adressée aux évêques, et où l'on remarque cette phrase: "Damnamus in perpetuum hunc libellum, mole exiguum, sed pravitate ingentem ». Le lendemain, Mgr Lartigue adresse à l'abbé Prince ces mots: "Comme La Parole d'un Croyant de Lamennais a été condamnée par une lettre du pape, je défends qu'à l'avenir on enseigne dans le collège de St-Hyacinthe rien des livres, des systèmes ou de la doctrine de cet auteur comme tiré de ses écrits. ${ }^{37}$

L'abbé Prince se croit obligé de se soumettre publiquement à l'encyclique Singulari nos du pape Grégoire XVI, publiée le 25 juin. Il envoie sa lettre au Canadien et à la Minerve, le 8 septembre. ${ }^{38}$ Un correspondant de la Minerve, qui signe le Bon

36 La Minerve, 21 août 1834.

3730 août 1834. RAPQ, (1943-44), 272. le 15 .

38 Elle parut dans la Minerve le 11 septembre 1834 et dans le Canadien 
Chrétien, accueille mal cette soumission. Et le voici parti en guerre contre un autre correspondant qui signe, lui, le Vieux Chrétien, et se porte à la défense de l'enseignement de la philosophie de La Mennais. Selon lui, ainsi l'écrit-il dans une première lettre, «l'encyclique de Grégoire XVI ne concerne en aucune partie le collège de St-Hyacinthe ${ }^{39}$; il ajoute dans une seconde lettre: «J'ai relu l'Encyclique du Saint Père et je persiste dans mon opinion; le système philosophique de l'abbé de La Mennais, n'est que l'objet d'un blâme secondaire, qui n'eut point eût lieu, si les paroles d'un croyant ne fussent venues réveiller les sollicitudes de l'autorité papale. ${ }^{40}$

Ces dernières querelles n'eurent pas de suite, sinon celle d'assombrir la réputation du collège de Saint-Hyacinthe et d'affecter la santé des principaux artisans du collège. Choquette nous cite une lettre de l'abbé Prince à ce sujet:

Je suis peiné, écrit-il, que Mgr de Québec ait des défiances contre nous. Il est vrai, j'ai bien des fautes et des péchés qui peuvent attirer l'orage sur le collège de St-Hyacinthe, mais mes péchés sont plutôt contre Dieu que contre les hommes, et après le meilleur examen, j'ignore absolument ce que j'ai pu commettre contre aucun collège du pays ou contre Mgr de Québec... Je ne puis me reprocher d'autre contravention à Mgr Signay que celle de mon adhésion publique à l'Encyclique de S.S. Grégoire XVI, car Sa Grandeur m'avait défendu (quoique je ne m'en mêlasse guère) d'écrire sur les gazettes. ${ }^{41}$

La réaction ou soumission de l'abbé Sabin Raymond se manifeste plus âpre. Grand admirateur de Lacordaire, il suit son exemple. Henri Lacordaire s'était séparé de son maître par ses « considérations sur le système philosophique de M. de Lamennais. ${ }^{42}$ Le disciple canadien prépare un «communiqué de vingt-sept pages in-folio destiné à La Minerve ».

39 La Minerve, 25 septembre 1834.

40 Ibid., 13 octobre. Cette réaction canadienne à propos de la condamnation de la théorie du sens commun se rapproche d'assez près de la réaction qui s'est produite en France.

41 Choquette, op. cit., I: 168.

42 Paul Vulliaud, Les Paroles d'un Croyant «de Lamennais》, (Amiens, Malfère, 1928), 139-144. 
L'article est virulent; rien n'apparaît de la façon généralement doucereuse de l'auteur. Mais on perçoit tout de même à travers l'anathème, qu'il ne peut se départir d'un fonds d'admiration pour le grand homme: "Alors, écrit-il, du sein de la France s'éleva ce cri de liberté qui, répété si promptement dans une grande partie de l'Europe, a fait tressaillir dans son retentissement jusqu'aux rives opposées de l'Océan ... sa plume superbe humilie la raison..., ses succès exaltant sans mesure en lui la conscience d'ailleurs si profonde de sa force et l'énergie si puissante de son âme il aspira à l'absolutisme, dans le domaine des intelligences; il voulut être le Napoléon de la pensée. ${ }^{43}$

Mgr Lartigue conseille la discrétion à l'abbé Raymond: « Vous n'êtes pas chargé de réfuter Le Canadien. Votre orthodoxie n'est pas en cause. Le Collège n'a pas soutenu à ma connaissance ce qu'il pouvait y avoir de faux et d'exagéré dans le système philosophique de Lamennais; et quand il l'aurait fait, il aurait soutenu ce qui n'était pas encore condamné. ${ }^{44}$

Quant à l'abbé Désaulniers, il revint à St-Hyacinthe à la fin de juin 1834, bien désintoxiqué de toutes les doctrines de La Mennais; il les combattit «avec la même ardeur qu'il mettait à les défendre avant son départ ». Il fit plus. Il sera celui à qui nous devons l'honneur d'avoir introduit le thomisme dans notre enseignement philosophique. ${ }^{45}$

Mais la semence jetée durant les années 32-34 devait germer. L'influence du La Mennais de l'Essai était terminée. Mais au même moment débute celle du Lamennais des Paroles d'un Croyant.

THOMAS Matheson, Licencié es-lettres (histoire) de l'Université Laval, Québec.

43 Choquette, op. cit., I : 168.

$44 \mathrm{Mgr}$ Lartigue profita de l'occasion pour faire disparaître tous les numéros de l'Avenir que possédait le Collège de St-Hyacinthe. Lartigue à Prince (Montréal, 8 novembre 1834), RAPQ, (1943-44), 280.

45 Groulx, L'enseignement français au Canada, I: 196. 